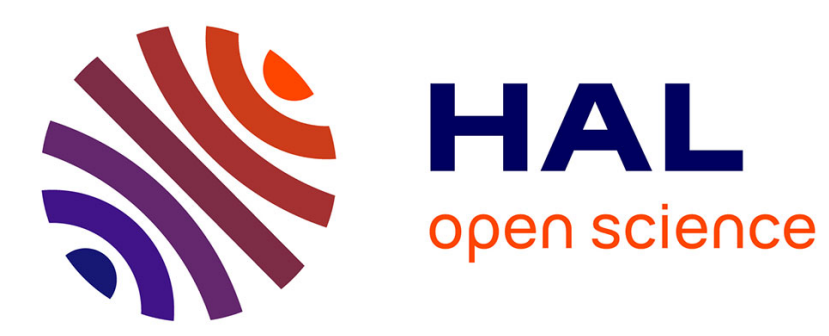

\title{
Human Spatial Navigation via a Visuo-Tactile Sensory Substitution System
}

Hervé Segond, Déborah Weiss, Eliana Sampaio

\section{To cite this version:}

Hervé Segond, Déborah Weiss, Eliana Sampaio. Human Spatial Navigation via a Visuo-Tactile Sensory Substitution System. Perception, 2005, 34 (10), pp.1231-1249. 10.1068/p3409 . hal-03041469

\section{HAL Id: hal-03041469 \\ https://hal.science/hal-03041469}

Submitted on 4 Dec 2020

HAL is a multi-disciplinary open access archive for the deposit and dissemination of scientific research documents, whether they are published or not. The documents may come from teaching and research institutions in France or abroad, or from public or private research centers.
L'archive ouverte pluridisciplinaire HAL, est destinée au dépôt et à la diffusion de documents scientifiques de niveau recherche, publiés ou non, émanant des établissements d'enseignement et de recherche français ou étrangers, des laboratoires publics ou privés. 


\title{
Human spatial navigation via a visuo-tactile sensory substitution system
}

\author{
Hervé Segond, Déborah Weiss \\ Unité de Recherche en Psychologie EA 3071, Faculté de Psychologie et des Sciences de l'Éducation, \\ Université Louis Pasteur-Strasbourg I, 12 rue Goethe, F 67000 Strasbourg, France; Conservatoire National \\ des Arts et Métiers (CNAM), 2 rue Conté, F 75003 Paris, France; \\ e-mail: herve.segond@psycho-ulp.u-strasbg.fr
}

\section{Eliana Sampaio}

Conservatoire National des Arts et Métiers (CNAM), 2 rue Conté, F 75003 Paris, France

Received 1 July 2002, in revised form 4 October 2004; published online 23 September 2005

\begin{abstract}
Spatial navigation within a real 3-D maze was investigated to study space perception on the sole basis of tactile information transmitted by means of a tactile vision substitution system' (TVSS) allowing the conversion of optical images-collected by a micro camera-into 'tactile images' via a matrix in contact with the skin. The development of such a device is based on concepts of cerebral and functional plasticity, enabling subjective reproduction of visual images from tactile data processing. Blindfolded sighted subjects had to remotely control the movements of a robot on which the TVSS camera was mounted. Once familiarised with the cues in the maze, the subjects were given two exploration sessions. Performance was analysed according to an objective point of view (exploration time, discrimination capacity), as well as a subjective one (speech). The task was successfully carried out from the very first session. As the subjects took a different path during each navigation, a gradual improvement in performance (discrimination and exploration time) was noted, generating a phenomenon of learning. Moreover, subjective analysis revealed an evolution of the spatialisation process towards distal attribution. Finally, some emotional expressions seemed to reflect the genesis of 'qualia' (emotional qualities of stimulation).
\end{abstract}

\section{Introduction}

We studied the capacities of navigation of blindfolded sighted subjects using a visuotactile sensory-substitution device. The study focused not only on the capacities for shape recognition and depth-cue perception, but also on the conditions that allow spatialisation genesis. The latter is the phenomenon of externalising the percepts, that is, locating subjective objects in extracorporal space.

Navigation is defined by the capacity of moving oneself throughout the physical world by using spatial information. It is a fundamental element of our perceptive-cognitive repertory. In everyday life, spatial navigation is very often required - whether or not in a familiar environment. It is studied in man and animal alike, within the framework of maze exploration (eg see the review by Van Horn et al 1998). In the case of visual deprivation, this activity is greatly impeded, although some blind subjects have developed a capacity for obstacle detection by means of echo location or 'facial vision'. This consists in the subject using acoustic information in the echoes-which can be defined by the reflection in the environment of the sounds produced by the subjects (eg Ashmead et al 1989; Arias 1996; Blash et al 1997; Silverstone et al 2000). Vision plays a prevailing part in space recognition and motion tracking within a 3-D environment. It has been very often observed that blind subjects suffer from alteration and delays when walking (Sampaio 1989a), primarily resulting from a feeling of insecurity (such as fear of obstacles), and this causes alteration of mobility - defined as the capacity of moving oneself in total security, comfort, grace, and autonomy in the environment (Foulke 1982). Since the invention of the white cane and the Braille system, there has been a significant evolution in noninvasive sensory-substitution devices (Warren and Strelow 1985). They have been designed 
to give blind subjects access to visuospatial information via sensory modalities other than vision. The main characteristics of this scientific, technological, and technical evolution are the use of computer science on the one hand, and the use of miniaturisation and non-magnetic materials on the other hand, the latter offering the opportunity for joint studies of both the behavioural and the cerebral activation level.

Today, two major categories of sensory-substitution systems prevail. The first are visuo-auditory systems. They collect information concerning location and distance of objects and, to a lesser degree, pick up information about the structure of objects (Kay 1980; Sampaio and Dufier 1988; Sampaio 1989b; Farcy et al 1997; Arno et al 1999). The second are visuo-tactile systems, which are used for providing optical information concerning object shape and depth (such as perspective and parallax) and, to a lesser degree, provide information about object distance and location (Bach-y-Rita et al 1969; Sampaio et al 2001).

With both congenitally blind and blindfolded sighted subjects using such systems, the central nervous system-ie cerebral and functional plasticity - is assumed to be adaptable. Broadly speaking, sensory-substitution systems, as they are called, translate stimuli which are specific to a sensory modality (such as vision) into stimuli specific to another modality (such as touch).

The use of such systems implies that the brain has the ability to learn how to interpret optical information about the environment, even though this information is carried via a non-visual sensory system (in the tactile vision substitution system, or TVSS, such information is carried via the skin).

Moreover, as Bach-y-Rita (1972) contended, "You do not see with your eyes. You see with your brain"; perception via a given sensory modality does not entirely depend on the sensations produced by it. Visual perception is in fact defined by any perception dealing with visual information, whether it is received by the retina and the cortico-visual system - which thus generates visual-like sensations - or by a TV camera connected to a specific device generating tactile sensations. Regarding this specific case, the TVSS makes it possible to convert visual images - detected by a miniaturised TV camera - to tactor-surfaced tactile images. In the 1960s, Bach-y-Rita's team, at the Smith-Kettlewell Institute of Visual Science, California, began developing the first version of the device, with the goal of helping persons who suffer from sight deprivation-whether congenital or not. The very first TVSS version contained a 400-tactor plate mounted on the back of a chair and connected to a TV camera. Bright images were detected by the TV camera and transformed into electric pulses which activated the tactors (piezoelectric vibrating tips). The subject was seated on the chair, with his/her skin in contact with the tactor plate, and handled the camera. Without any training, the subjects-blind and blindfolded sighted alike-were able to perceive some simple targets, to turn towards them, to discriminate horizontal, vertical, or curved lines, and to point out the directions of the moving targets. Geometrical shape identification required a training period (Bach-y-Rita 1972).

Nevertheless, the most remarkable phenomenon about the utilisation of the TVSS subsequent to intensive training - was that the ability to recognise shape was accompanied by an externalisation of percepts into objects in space. The subjects' subjective reports reveal that, at the beginning, the stimulations could be felt only through the skin. But after training for perception, the subjects' sensations related to touch were replaced by the perception of objects situated at a distance in front of them.

The techniques developed in the 1960s and tested in the 1970s had a surprisingly limited impact on the everyday life of both the blind and partially sighted people. The devices were bulky, noisy, and power hungry. Since then, the TVSS has undergone several technological improvements. The main ones are: first, miniaturisation; second, optimised comfort in the utilisation of the tactile interface-owing to electrotactile 
stimulation; and, last, an improvement in image resolution. Furthermore, with the new versions of the TVSS, the matrix containing the tactile stimulation has been placed on the forearm, abdomen, forehead, thigh, and fingertip, and more recently on the tongue, after previously having been placed exclusively on the back. The subjective localisation of the information transmitted by the TV camera remained in the 3-D space in front of the subjects, regardless of the parts of the body stimulated. Among the versions of the TVSS, the ETV 4 (http://www.forethoughtdevelopment.com/videotac.html) has been used for the present research. The basic principles of the device have been retained. The device comprises a data-processing container designed for converting visual images, collected by a TV camera - or programmed by a computer-into tactile images transmitted via a 96-tactor matrix (12 lines $\times 8$ columns; see figure 1$)$.
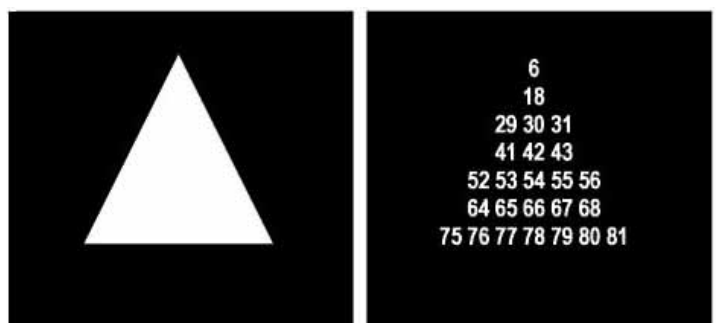

Figure 1. Example of a 'tactile image' (on the right), such as it appears on the monitor of the TVSS, with the corresponding visual information collected by the camera, such as it is also presented on a monitor screen (on the left). Numbers on the TVSS screen materialise each activated tactor (number ranging between 1 and 96, relating to its position on the matrix of 96 tactors).

Although the devices have been significantly improved in technological respects, knowledge about perceptual mechanisms has not been fully explored. Yet, the advantages of such an approach for the blind are obvious. Bach-y-Rita and his collaborators emphasise the significant role of the subjects' action in the exploration of their environment with a sensory-substitution device - in particular by handling a camera (White et al 1970). The importance of movement for perception is basic for ecological psychology as initiated by Gibson (1966, 1979), who considered reciprocal relations between perceptual and motor activities and the existence of a perception-action system with perceptual-motor links, where sensory and motor units are functionally integrated, and not separated, as they had first been considered. More recently, Lenay et al (2000) stated that recognition of perceived objects is based on the detection of perceptivemotor invariants, indicating a search by the action of the subjects and the mental construction of constant connections between action and feeling. Lenay et al thus think that spatial location, as well as shape recognition, correspond to temporal syntheses of the successive feelings according to a rule connecting action with feeling.

We tested these capacities by proposing a navigation task throughout the physical world based exclusively on visuo-tactile substitution information. Some studies were run in order to test spatial navigation abilities (Jansson 1983). But they essentially demonstrated, in a small number of subjects, the capacities of walking towards a target pole, detecting obstacles, and moving according to certain rules in relation to these obstacles (vertical bars). Jansson (1983) conducted a set of experiments in a tactually guided slalom walk, using a $12 \times 18$ matrix of vibrators on the forehead (Electrophthalm system), in which the most complex situation comprised a $6 \mathrm{~m}$ path with one target and two obstacles - each consisting of a $4-\mathrm{cm}$ wide vertical pole. In this very simple environment, one blind subject succeeded in carrying out the task on the sole basis of obstacle detection after a preliminary training, despite some collisions with obstacles.

We aimed to propose a more complex navigation task in the 3-D space, in which the subjects had to find the way out not only by detecting the presence or absence of obstacles, but also on the basis of a perceptual analysis of directional cues, optical information about depth, and the generalisation capacities in recognising these cues from different points of view (ie perspective). 
The use of mazes, designed to apprehend the fundamental capacity to move, has had a significant history in neuropsychological literature and clinical framework (see the review by Van Horn et al 1998). The study of cerebrally impaired patients, in particular, provided extensive data on cognitive capacities involved in this task. In the maze-based test, four major functional systems have usually been studied. Each of them is associated with particular cerebral areas such as sensory discrimination, spatial memory, execution function, and decision motor-execution (Flitman et al 1997). Changes in any one of these functional systems affect the performance within a maze.

According to Beritoff, an organism is capable of space orientation-required for this kind of task - when it is able to find the position of the objects and project this position in relation to the other objects and itself. He makes the assumption that, from these perceptions, images of the repartition of the objects in the surrounding world can be created (Beritoff 1965; quoted by Berthoz 1997).

The basic questions such as: "where am I?", "where are the other elements of the surroundings?", "by what means can I go somewhere?", reflect the functions of the navigation system. Human subjects are presented with this kind of task in the laboratory. It resembles the tasks traditionally used to train other animal species (Morris 1981) to acquire space skills. Recent developments of data-processing software have made it possible to simulate a virtual maze-type environment, thus allowing a very valuable measurement of human navigation abilities in an environment very similar to reality. When navigating in a virtual maze-according to visual information-the subjects have a strong feeling of 'presence'. Here, the word 'presence' expresses the subjective experience of being somewhere while being physically elsewhere (Held and Durlach 1992; quoted by Maguire et al 1999). Witmer and Singer (1994; quoted by Maguire et al 1999) have identified a significant correlation between 'presence' and the performance undertaken in a virtual environment.

For the exploration task, we proposed a truly 3-D environment rather than a virtual maze. Indeed, very few research studies to date have pondered the meaning of the results obtained about the perception of 3-D objective reality-based on situations in which the subjects had to visualise depth from 2-D depth representation-in other words, from a missing dimension. Griffin has worked on various modes of orientation of birds, and has distinguished 'piloting' - the search and the use of familiar reference marks in order to reach a goal-from 'navigation' - a mechanism allowing them to go from an unknown spot to a known goal (Griffin 1942; quoted by Durup 1985). The geographical representations at stake in the proposed navigation task can also be called piloting tasks - if one refers to Griffin's work. These geographical representations can be integrated into a route connecting the landmarks with one another and providing the decision sequences at the point of choice (eg turning to the left). Such a path should lead to a representation of sensorimotor sequences proceeding from a starting point to a finishing point, independent from the route (Lepecq and Pêcheux 1985).

To date, the ETV 4 version of the TVSS used in this experiment does not allow much mobility (connections exist between the matrix and the computer interface). Consequently, we proposed a navigation task in which the subjects had to control by radio a mobile robot (see section 2.2.2), equipped with a camera, placed inside a maze. Subjects could perceive sensory and perceptual consequences of their actions by manipulating two joysticks. Thereby, a sensorimotor coupling could be performed from the tactile information provided by the matrix and visual cues located in the maze. The same navigation task was then used to compare the navigation capacities of blindfolded sighted subjects, who navigated on the sole basis of visuo-tactile information with the efficiency of sighted subjects who made the same navigation task under visual control by watching the monitor system of the TVSS (see section 4.5). 


\section{Method}

\subsection{Subjects}

Eight healthy, sighted subjects (four women and four men) aged 22 to 40 years, participated in this experiment. The average age was 28 years for women, and 34 years for men.

\subsection{Apparatus}

2.2.1 The tactile vision substitution system, ETV 4 version. This device is composed of four elements: a micro TV camera, a data-processing system, a converter container, and a 96 micro-electrode matrix, $7.5 \mathrm{~cm}$ by $9.5 \mathrm{~cm}$, placed on the subjects' abdomen (directly above the navel). The principle on which it works comprises three stages. The first consists of decomposing the visual images, the second of translating them into pulses, and the last one in distributing them to all of the 96 micro-electrodes of the matrix. The temporal development and the physical form of the electric pulses are parameterised according to five criteria: intensity, width, rest interval, number of pulses, and burst period. The relative values of these criteria in relation to one another, and the concomitant variation when the camera is shifted, determine the fullness and quality of the function of the TVSS (for a detailed description, see Aiello 1998). The electrical energy generated by the impulse and transmitted to the touch receptors is proportional to the surface of the wave. The skin is first cleaned with alcohol and coated with a scanning-like conductive gel. The tactile-somesthesic system is stimulated by 'tactile images' with a $32 \mathrm{~Hz}$ vibration frequency and a $21.56 \mathrm{~V}$ pulsation amplitude.

2.2.2 The remote-controlled mobile robot. Both the mobile robot (which we named the 'mobile sensory-motor coupling', MSMC; see figure 2), and the TV camera mounted on it, are remotely controlled by high-frequency connections. This allows them full movement. The MSMC was especially designed for this experiment. It is $23 \mathrm{~cm}$ long, $14 \mathrm{~cm}$ wide, and $17.5 \mathrm{~cm}$ high. The camera, with a $120^{\circ}$-sight-angle lens mounted on it, is at the front of the robot. It collects the visual information in the maze and transmits it to the TVSS in order to display it on the electro-tactile matrix.

Subjects used two joysticks. The first enabled them to control vertical scanning of the camera in order to perform fuller discrimination of visual stimuli and cues. The second enabled them to control movements of the MSMC by three means: forward movements, backward movements, and rotations in either direction by the front wheel turning at an angle of $160^{\circ}$.

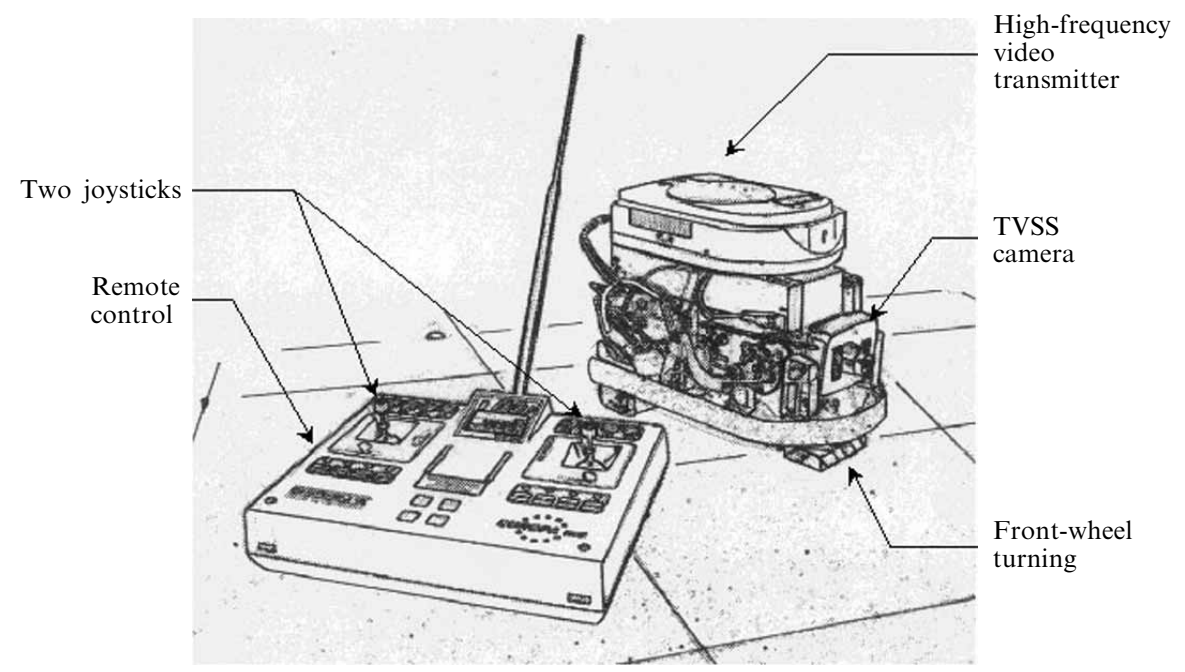

Figure 2. The mobile robot of sensory-motor coupling with a frontal TVSS camera mounted on it, and the remote control with two joysticks. 
2.2.3 A truly 3-D maze. A truly 3-D maze of $3 \mathrm{~m}$ by $3 \mathrm{~m}$ was oriented, designed for, and adapted to the characteristics of the size and mobility of the robot. It comprised a starting room, A, a finishing room, B, and eight rooms in between (see figure 3). Room A was large and caused few spatial constraints. The subjects could easily acquire coordination skills to control the robot in relation to the camera movements and thereby carried out a sensory-motor coupling. Room B and the eight eight-sided middle rooms were smaller. The robot was nonetheless able to explore the various cues on the walls. The rooms were connected to one another by corridors with lines on the floor. Inside the maze, there were eighteen 'saloon'-type double doors. White triangular cues were placed on these doors. Because of the limited size of the maze, cues also featured on fake doors. The whole maze was black, in order to facilitate contrasted perception of white cues.

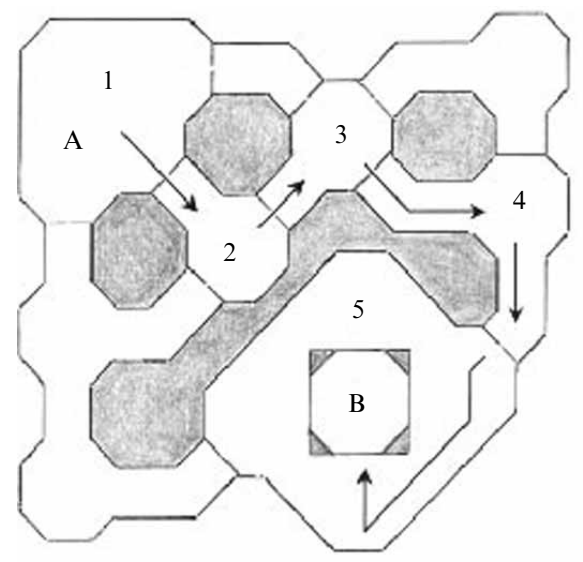

(a)

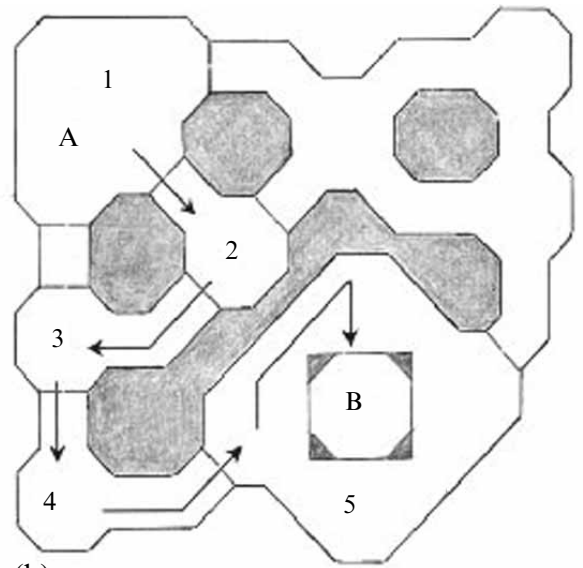

(b)

Figure 3. Maze plan, decomposed into 5 zones, from the starting room, A, to the arrival zone, B, for the two routes. (a) Route 1. (b) Route 2.

Along this route, from room $\mathrm{A}$ to room $\mathrm{B}$, the subjects had to make a series of choices in order to find the right path, composed of five exploration zones of a varied configuration. Decomposing the route into 5 zones offered the researchers two advantages: performance evolution among the zones could be analysed in a single exploration on the one hand, and between two navigations on the other hand (the subjects had to take one route during the third session and another route during the fourth session-see below). Each zone comprised an exploration room. In zone 1, there was a large starting room. Zones 2, 3, and 4 contained one eight-sided middle room each. In zone 5, the finishing room was of more complex configuration. All the rooms were connected with one another by a network of corridors. Throughout this route, the subjects were confronted by three increasing difficulty levels-related to the motion constraints of the robot. Difficulty level 1 was represented by zone 1. Manoeuvering and positioning the robot was relatively easy and helped the subjects to improve cue recognition. In addition, the large space in zone 1 facilitated the exploration, which was of a circular type. In difficulty level 2-in zones 2, 3, and 4-restricted space increased the exploration difficulty. Being guided by the white line on the floor, the subjects had to control the robot and guide it into the corridors, as the robot had less and less space to move backwards from the triangular cues. Difficulty level 3-in zone 5represented the highest complexity level. The subjects had to follow a corridor delimited by walls belonging to two overlapping rooms. Because the MSMC had a restricted space for navigation, the subjects' aids were perspective cues, such as vanishing lines horizontally placed on the walls and triangular cues generally perceived at an angle. 
2.2.4 Spatial cues. Two types of cues were available to help the subjects make choices to find their bearings in the maze. The first category consisted of a continuous white line on the floor and at the centre of the corridors - as a landmark - and another line placed horizontally on the room walls. The second category consisted of 2-D triangular cues (isosceles triangles) on the doors. Thanks to the orientation of the cues, the subjects were informed whether they could cross the doors; if not, they were informed about the direction they had to follow (see figure 4).

Floor and lateral lines were solely a navigation aid, but the triangular cues had to be explored and discriminated by the subjects in order to succeed in the task.

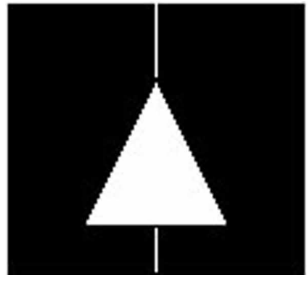

Move ahead

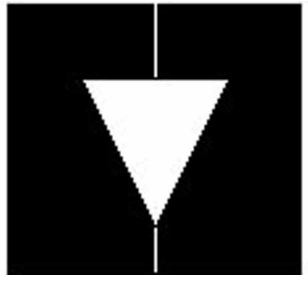

Move backwards

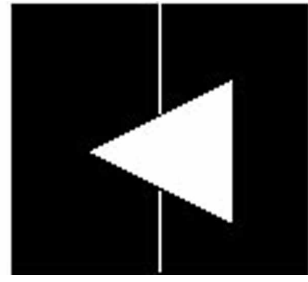

Move leftwards

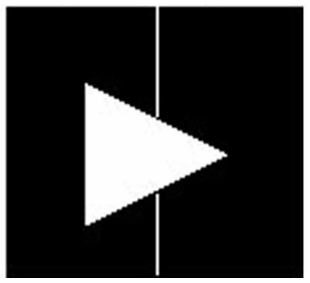

Move rightwards

Figure 4. Two-dimensional triangular cues used on the doors to indicate the direction to follow.

\section{Procedure}

Four sessions were used. Their average duration was $2 \mathrm{~h}$ each, and the average interval between them was 3.7 days $(\sigma=1.8)$, according to the subjects' availability. The first session included a single phase of 'familiarisation/assessment' for 2-D triangular-cue identification. The next three sessions comprised a phase of 'familiarisation/assessment', followed by the actual navigation phase. In the second session, navigation was carried out thanks to visual information, via a computer screen related to tactile information, via the TVSS matrix, whereas in the third and fourth sessions only tactile information was available. Subjects were blindfolded during all phases and sessions except the navigation phase in session 2 .

Throughout the experiment (all four sessions), subjects were seated at a table in front of the two-joystick remote control and wore the TVSS matrix on their abdomen (cf figures 5, 6, and 7). During the familiarisation phases, aided by the joysticks of the remote control, the subjects had to explore 2-D triangular cues on a blackboard in front of them (see figure 5). The camera mounted on the robot collected visual information and transmitted it to the TVSS array - and to the computer screen during the

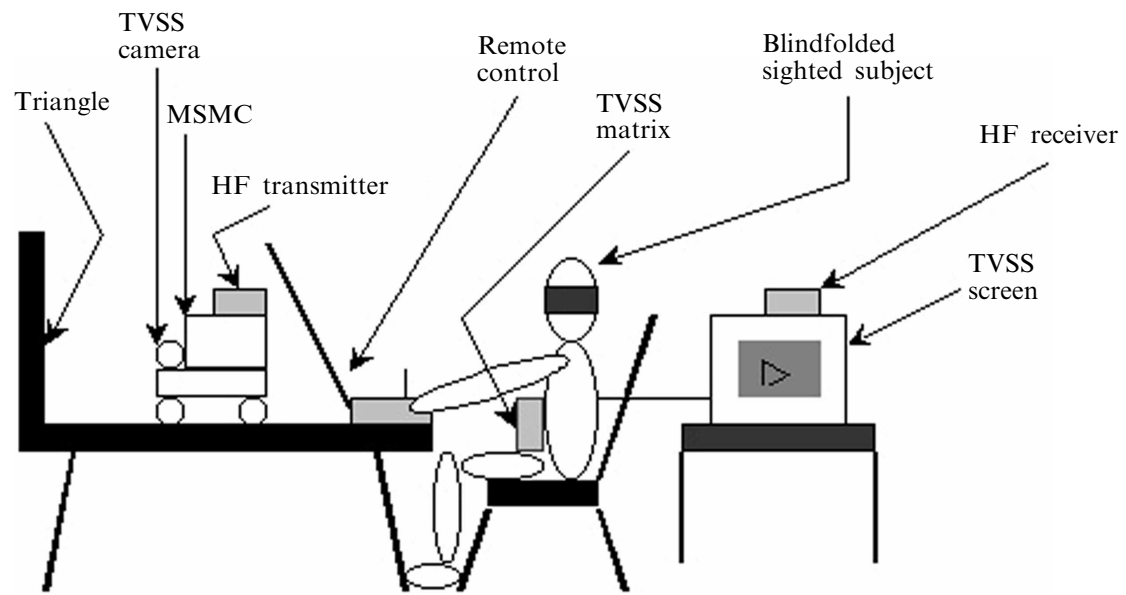

Figure 5. Experimental set-up during the familiarisation/assessment phase, in the four sessions. 
navigation phase in session 2. During familiarisation phases, the robot was mounted on a table, between the subjects and the blackboard. The camera was guided towards the triangular cues placed $30 \mathrm{~cm}$ away from the camera. In this condition, with the robot remaining stationary, only the TV camera could be controlled via the remote control (see figure 5). The robot was also placed in the maze for the navigation phases. Remote control was then used to shift it and to position the camera (see figures 6 and 7).

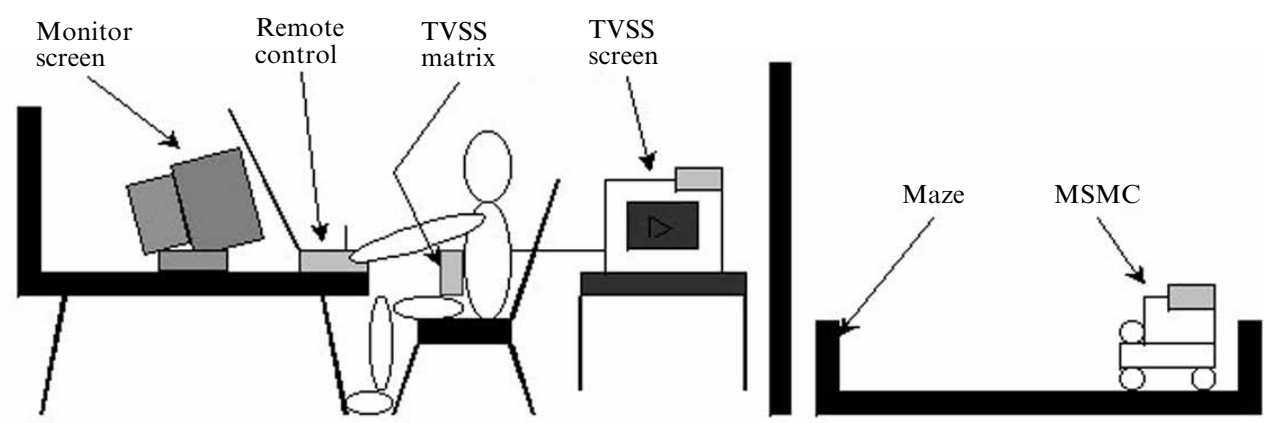

Figure 6. Experimental set-up during the navigation phase in the second session.

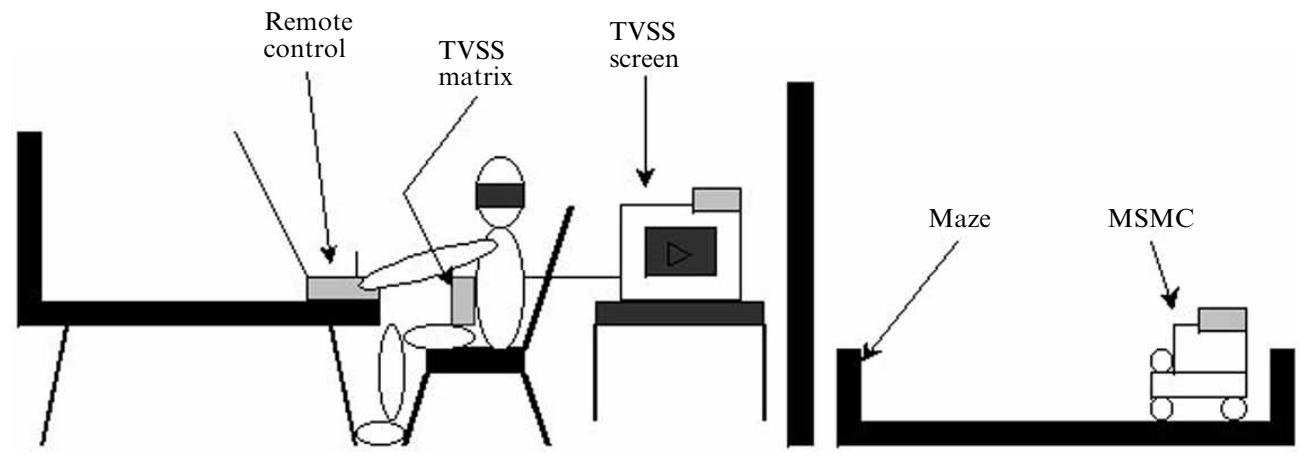

Figure 7. Experimental set-up during the navigation phase for sessions 3 and 4 .

\subsection{Session 1: Familiarisation with the stationary mobile}

The object of the first session was to set a baseline for triangular 2-D cue-discrimination capacity, and to familiarise subjects with the handling of the camera controls and with the feelings of TVSS-based stimulation related to the four possible orientations of an isosceles triangle (upwards and downwards, right and left).

The method used to determine the stimulation intensity most comfortable for subjects consisted of constantly increasing the stimulations in ten-unit stages from a ninety-unit PA infraliminary value. Once this had been done, session 1 comprised three stages. During the first stage, subjects were exposed to TVSS stimulations-each of them was exposed to each of the four triangle orientations-and the experimenter himself controlled the camera and drew the subjects' attention to tactile information useful for discrimination (stimulation intensity and the effects on the sensory level generated by camera scanning).

During the second stage, subjects themselves drove the camera using the joystick, and freely explored the four orientations in order to note sensory-motor correspondences.

Finally, during the third stage, the researcher assessed subjects' discrimination capacity when presented with four blocks of four orientations - displayed twice eachin a pseudo-random way (ie thirty-two trials). Subjects were asked to explore the cues via the camera, and then to indicate which category of visual information the perceived tactile stimulations corresponded to. Subjects were also asked to provide a confidence index of 0 to $3-0$ for 'random', 1 for 'unsure', 2 for 'relatively sure', 3 for 'absolutely 
sure'-for each reply. In order to facilitate training, subjects were also informed that they would be given a feedback following each reply and that they were allowed to re-explore cues in case of any wrong reply.

\subsection{Session 2: Introduction to tactually and visually controlled navigation}

The object of session 2 was to check whether there was an improvement in 2-D cue discrimination in comparison with session 1, and also to introduce subjects to the navigation task which was to be carried out in sessions 3 and 4 , by facilitating it as much as possible. This task comprised two unusual conditions and was performed via a TV camera placed on a remote-controlled robot, and via an artificial and totally new sensory modality, by the mediation of the TVSS.

The triangular-cue familiarisation phase was carried out in the same conditions as in session 1, with the exception of one condition: in session 1, subjects underwent thirty-two trials, whereas starting with session 2, trials were to be carried out until an $80 \%$ success criterion of correct replies was met. If such a criterion could not be met, subjects would have to undergo all of the thirty-two trials. In such a case (which, in fact, never occurred), there would have been additional training sessions until this success criterion had been met. In addition, subjects were told that, although their exploration was timed, it was more important to provide right replies.

The navigation phase was made up of two stages. In the first stage, subjects had to become familiar with controlling the robot inside the maze, under visual control. This navigation was to take place following the first route, which comprised three cue categories. The first category consisted of on-the-door triangular cues indicating which direction, and possibly which door, had to be taken. The second consisted of lateral stripes on the walls indicating that the robot was facing an obstacle. When discontinuous, these stripes let subjects know that the robot was close to a cue. The third category was a continuous line on the floor along the corridors, which enabled subjects to control the robot along the corridors from room to room. Whenever a line ended, subjects thereby knew the robot had entered a room. Subjects were asked to go from the starting room, A, to the finishing room, B, by using the indications they obtained through the cues perceived on a video screen in front of them (non-degraded images). During the second stage, the task was the same with an identical route; however, this time, tactile stimulation provided by the TVSS was combined with visual information.

\subsection{Session 3: Testing phase regarding navigation via the TVSS}

After another 'familiarisation/assessment' phase with triangular cues-identical to the previous one-had been carried out, blindfolded subjects were asked to perform a navigation task. Here, they could use only tactile information provided by the TVSS. The route and the cues were the same as in the previous session. In order to succeed in the task, only the triangular cues had to be explored. Twenty-four triangular cues were placed along the route. Subjects had to explore at least ten cues in order to determine which route they had to follow (see section 3a).

Subjects were provided with feedback whenever they wished, with the following conditions. First, the feedback had to help to determine how far the robot was from the cues, if the cues were well centred relative to the robot, and which cue category they were exploring (triangular cues, lines on the floor, or lateral stripes). Subjects were not provided with an indication about the direction they had to take nor about the orientation of triangular cues they discriminated. Second, the feedback was aimed at providing information on whether the robot was able to go forward or backward (eg when it was stuck against a wall or when it was impossible for it to go back). During this navigation task, subjects were asked to voice their impressions as extensively as possible. They had to relate, in particular, what they perceived and what decisions resulted from it. Their voice was recorded. 


\subsection{Session 4: Testing phase regarding navigation via the TVSS; route 2}

This session was performed in the same way as the previous one in terms of both 'familiarisation/assessment' and navigation phases. However, it was carried out following a new route, totally unknown to the subjects. The two routes were not counterbalanced within the experiment for technical and practical reasons. Indeed, changing the route required a complex adjustment of the maze. Nevertheless, the second route had the same complexity level as the route in the previous session. The complexity level took account of both the overall route configuration and all of the zones that constituted these routes (see figure $3 b$ ).

Once the subjects had completed sessions 3 and 4, they were subjected to an interview with the aim of analysing the spatial perception they had experienced. The value of movements connected to perception, in particular, was studied. So was the informative nature of the various cues for visual space perception via the TVSS. This phenomenological-type approach allowed the researcher to follow the way subjects perceived the space they explored from their own point of view.

\section{Results}

Subjects' performance was analysed according to the following four criteria:

(i) The rate of correct replies regarding 2-D triangular-cue discrimination, during the four familiarisation/assessment phases, and during navigation tasks in sessions 3 and 4 (and routes 1 and 2).

(ii) The average cue-exploration time, during the last three familiarisation/assessment sessions, and during the last two navigation sessions, zone by zone, and including both routes.

(iii) The time it took the subjects to go through the five maze zones in the last two navigation sessions. The average duration of subjects' presence in each zone per square metre was calculated - the surface of every zone being set to the same scale - a $1 \mathrm{~m}^{2}$ surface for all.

(iv) The analysis of subjects' replies to the question paper. The researcher could thereby determine when and how subjects externalised visual percepts while carrying out explorations of routes 1 and 2 in navigation sessions 3 and 4, respectively. These replies were expected to supply complementary elements in terms of quality as regards exploration strategies used in the maze. They were also expected to allow the researcher to draw conclusions about spatiality genesis from subjects' subjective experience. Spatiality can be defined as subjects' involvement in the space they explored and depth perception.

The main objective of this research was to check whether the navigation task based solely on visuo-tactile substitution information was feasible or not. This goal was achieved from the very first navigation task (in session 3). It appeared that all subjects did indeed succeed in this task. They were able to control the robot from the starting room to the finishing room, thanks to the orientations displayed by the triangular cues in the maze. As the task proceeded, performance gradually improved, so the experimenter supplied subjects with less and less feedback and commentaries. In fact, feedback and commentaries only consisted in encouraging the subjects in cases of need, and sometimes giving some information on driving if the subjects encountered some difficulties in extricating the robot from an obstacle. But the information given never concerned the route to be followed.

Overall, in spite of its lengthy duration and the encountered difficulties, most subjects treated the task like a game. On average, the first navigation (in route 1, session 3) was completed in $71.31 \mathrm{~min}(\sigma=23.84)$, and the second one (in route 2 , session 4$)$ in $64.69 \min (\sigma=4.99)$. However, the drop in navigation time observed between these two sessions was not significant $\left(t_{7}=0.88, p<0.4\right)$. Although subjects had not been familiarised with them, the horizontal and lateral stripes on the maze walls were reported by subjects to be good clues, as they allowed them to perceive space configuration. 


\subsection{Discrimination of triangle orientation during familiarisation/assessment and navigation phases}

Table 1 shows the evolution of performance in triangle-orientation discrimination in all four familiarisation/assessment phases. It also allowed us to compare the latter performance with the one carried out during navigation phases. Most frequent mistakes consisted in identifying triangular cues wrongly and confusing triangles with lateral stripes.

Overall, performance in discrimination of triangle orientation significantly improved between the second familiarisation/assessment session and the third. This was evidenced, as the $80 \%$-correct response criterion was fulfilled more and more rapidly between F2 and F3 (fifteen versus eleven trials; $F_{1,7}=7, p=0.033$ ), and the number of correct replies in session 4 was stabilised.

Correct-reply rates noted in familiarisation/assessment phases were scarcely different from one another, for these rates depended on the same criterion fulfillment (ie correct replies reaching $80 \%$ ).

Table 1. Average performance in discrimination of triangle orientations during familiarisation and navigation (and standard deviation) and average number of explored cues. F1 to F4 correspond to the four familiarisation/assessment sessions; T1 and T2 correspond to routes 1 and 2 in navigation sessions 3 and 4 respectively.

\begin{tabular}{|c|c|c|c|c|c|c|}
\hline & \multicolumn{4}{|c|}{$\begin{array}{l}\text { Average performance during } \\
\text { familiarisation/assessment } / \%\end{array}$} & \multicolumn{2}{|c|}{$\begin{array}{l}\text { Average performance } \\
\text { during navigation } / \%\end{array}$} \\
\hline & $\mathrm{F} 1$ & $\mathrm{~F} 2$ & F3 & $\mathrm{F} 4$ & $\mathrm{~T} 1$ & $\mathrm{~T} 2$ \\
\hline Correct replies & $\begin{array}{l}80.3 \\
(13.66)\end{array}$ & $\begin{array}{l}90 \\
(8.65)\end{array}$ & $\begin{array}{l}90.8 \\
(7.95)\end{array}$ & $\begin{array}{l}89 \\
(7.36)\end{array}$ & $\begin{array}{l}74 \\
(13.1)\end{array}$ & $\begin{array}{c}70.7 \\
(17.3)\end{array}$ \\
\hline $\begin{array}{l}\text { Number of } \\
\text { explored cues }\end{array}$ & $\begin{array}{l}32 \\
\text { (stated) }\end{array}$ & 15 & 11 & 11 & 20.3 & 20.4 \\
\hline
\end{tabular}

When we compared the correct-reply rate in the familiarisation phase with the correct-reply rate in the navigation phase, we noted that cue-discrimination performance decreased in navigation sessions 3 and 4, routes 1 and 2 in comparison with the last familiarisation/assessment session. An a posteriori analysis (Newman-Keuls test) indeed confirmed that correct reply rates between the navigation phase and the familiarisation/assessment phase preceding it decreased between F3 and T1 $(p=0.011)$; and between F4 and T2 $(p<0.001)$. The drop in performance may be explained by the fact that positioning the robot in relation to the targets generated constraints. During the navigation phase, owing to these constraints, subjects sometimes discriminated triangular cues even though the latter were not perfectly perpendicular to the camera axis. With the camera positioned at an angle to the targets, visual images projected onto the TVSS electro-tactile matrix were distorted. During the familiarisation/assessment phase, however, with the camera in the perpendicular axis of the stimuli and the stationary mobile, there was no such constraint. Nevertheless, it is noteworthy that, in spite of this drop, correct-reply rates were still satisfactory and far superior to randomcorresponding to a success rate of $25 \%$ for each of the four orientations $\left(\chi^{2}=816.22\right.$, $p<0.0001$ for $\mathrm{T} 1 ; \chi^{2}=750.97, p<0.0001$ for $\mathrm{T} 2$ ).

4.2 Choosing directions during navigation phase and exploration time per cue and per zone, regarding routes 1 and 2

4.2.1 Recognising triangular-cue orientations during the navigation phase. Table 2 represents the average correct-reply rates for recognition of triangular-cue orientation in each zone in the maze and in both routes. Figure 8 allows us to visualise the profiles of the evolution of correct-reply rates in both routes. 
Table 2. Comparison of performance regarding discrimination of four orientations of an isosceles triangle between routes 1 and 2 (ie navigation sessions 3 and 4). Standard deviation is shown in parentheses.

\begin{tabular}{lccccccc}
\hline Route & \multicolumn{6}{l}{ Orientation discrimination performance/\% } & \multirow{2}{*}{$\begin{array}{l}\text { Mean number of } \\
\text { explored cues }\end{array}$} \\
\cline { 2 - 6 } & zone 1 & zone 2 & zone 3 & zone 4 & zone 5 & average & \\
\hline 1 & 62.9 & 69.1 & 78.8 & 95.9 & 63.4 & 74 & 20.3 \\
& $(20.3)$ & $(29.4)$ & $(26.4)$ & $(11.7)$ & $(23.3)$ & $(13.1)$ & $(4.2)$ \\
2 & 55.9 & 72.6 & 70.9 & 85.5 & 68.5 & 70.7 & 20.4 \\
& $(25.8)$ & $(24.1)$ & $(26)$ & $(20.7)$ & $(17.1)$ & $(17.3)$ & $(6.0)$ \\
\hline
\end{tabular}

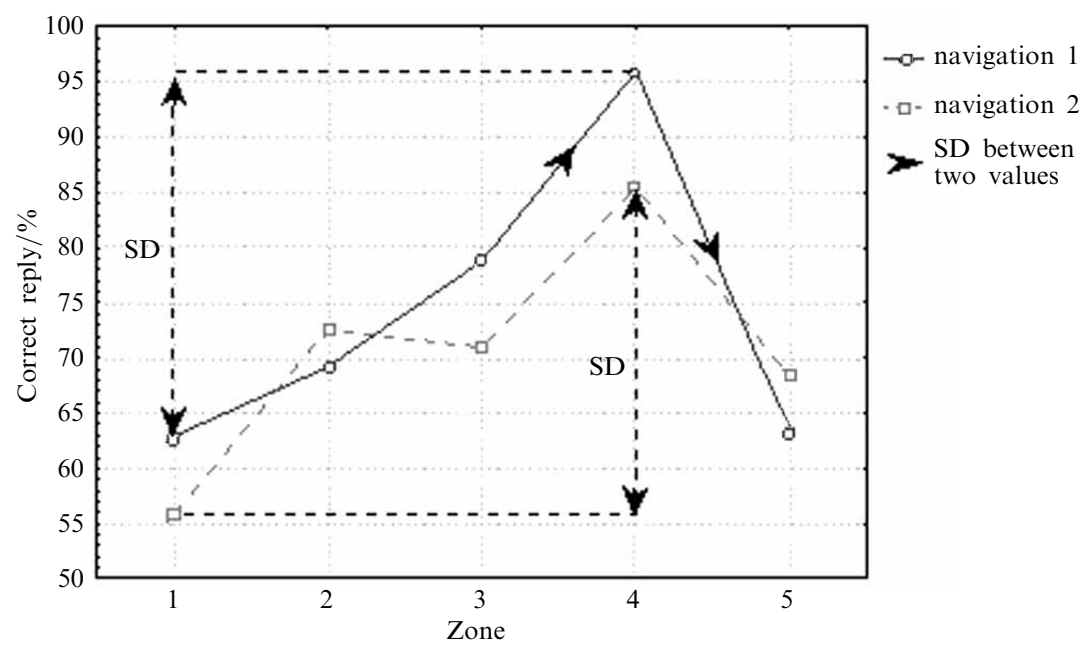

Figure 8. Evolution of success rates per zone in both route explorations (SD = significant difference).

Correct-reply rates were transformed into arc sines. The normality of distributions thereby obtained was checked empirically and confirmed by means of KolmogorovSmirnov statistical tests. So, we conducted a three-factor (multivariate) analysis of variance (MANOVA). The design was 2 (sex: men versus women) $\times 2$ (navigation: first versus second) $\times 5$ (zone: 1 to 5 ) mixed-factor MANOVA, in which sex was the between-subjects factor and navigation and zone were within-subjects factors. This analysis showed that there was no influence of sex on performance. During a second phase, male-related and female-related data were gathered and analysed together (see the above structure analysis in the sex factor). This analysis revealed a similarity in profiles of performance evolution from one zone to the next between navigations 1 and 2 . This similarity was revealed because the navigation factor exercised no effect $\left(F_{1,7}=0.317, p=0.59\right)$ and because the zones exercised a significant influence $\left(F_{4,28}=7.61, p<0.0003\right)$. In both navigations, this evolution was evidenced by an improvement in triangular-cue orientation discrimination between zones 1 and 4 (navigation 1: $F_{1,7}=32.3, p<0.0008$; navigation $2: F_{1,7}=13.376, p<0.009$ ). In addition, during navigation 2 , this improvement in performance appeared earlier than it did during navigation 1, for this significant rise in correct-reply rates was noted in zone $2\left(F_{1,7}=5.8, p<0.05\right)$. This phenomenon seemed to be a training effect between both navigations. It was noted that performance in the finishing room (zone 5) was of lower quality. This may be accounted for by the significant fall in performance between zones 4 and 5 that occurred during navigation $1\left(F_{1,7}=32.54, p=0.0007\right)$ and navigation $2\left(F_{1,7}=7.04, p=0.032\right)$. Zone 5 was the most complex spatial configuration of the maze. 
4.2.2 Exploration time per cue and per zone in routes 1 and 2. Table 3 shows the average durations of triangular-cue exploration per zone in navigation sessions 3 and 4 and routes 1 and 2 . According to the 2 (navigation) $\times 5$ (zone) ANOVA, in which navigation and zone were within-subjects factors, triangular-cue exploration time was not affected by these factors during the task. However, this result does not reflect the reality as the sizes and degrees of complexity in the different zones were not equivalent.

Table 3. Exploration time in each of the 5 zones for routes 1 and 2. Standard deviation is shown in parentheses.

\begin{tabular}{lcccccc}
\hline Route & \multicolumn{6}{l}{ Exploration time/min } \\
\cline { 2 - 7 } & zone 1 & zone 2 & zone 3 & zone 4 & zone 5 & average \\
\hline 1 & 18.9 & 52.8 & 22.1 & 12.4 & 12.9 & 23.9 \\
& $(12.4)$ & $(40.8)$ & $(15.2)$ & $(5.8)$ & $(2.5)$ & $(10.1)$ \\
2 & 9.1 & 28.4 & 23 & 15.1 & 20.4 & 19.1 \\
& $(5.3)$ & $(16.7)$ & $(13.5)$ & $(5.6)$ & $(10.2)$ & $(7.3)$ \\
& & & & & & \\
\hline
\end{tabular}

In order to neutralise the size disparity and thus to be able to determine whether a training effect occurred in terms of exploration time, the sizes of all zones in the maze were set to a single $1 \mathrm{~m}^{2}$ surface unit. This was the basis for calculating the exact surface of every zone taken during routes 1 and 2. The mobile exploration time in each of these zones-which were of non-equivalent surfaces-was then converted into a single exploration time of $1 \mathrm{~m}^{2}$ (ie for zone 1 , route 1 exploration time was $200 \mathrm{~s}$ for $0.81 \mathrm{~m}^{2}$, that is $246.9 \mathrm{~s} \mathrm{~m}^{-2}$, etc). Although this operation allowed us to compare exploration durations of zones of equivalent surface, one should not forget these zones were different in terms of difficulty level owing to their spatial configuration.

Once again, the three-factor analysis of variance (MANOVA) [2 (sex: men versus women) $\times 2$ (navigation: first versus second) $\times 5$ (zones) in which sex was the betweensubjects factor and navigation and zone were within-subjects factors] did not reveal any effect of sex on the collected data. The sex factor was pooled and the last analysis allowed us to bring to the fore an effect of the zones successively travelled through during exploration time $\left(F_{4,28}=7.09, p<0.0005\right)$. Thanks to Newman-Keuls-type a posteriori analyses, we were able to specify the nature of this performance evolution throughout the zones. This evolution is shown in figure 9.

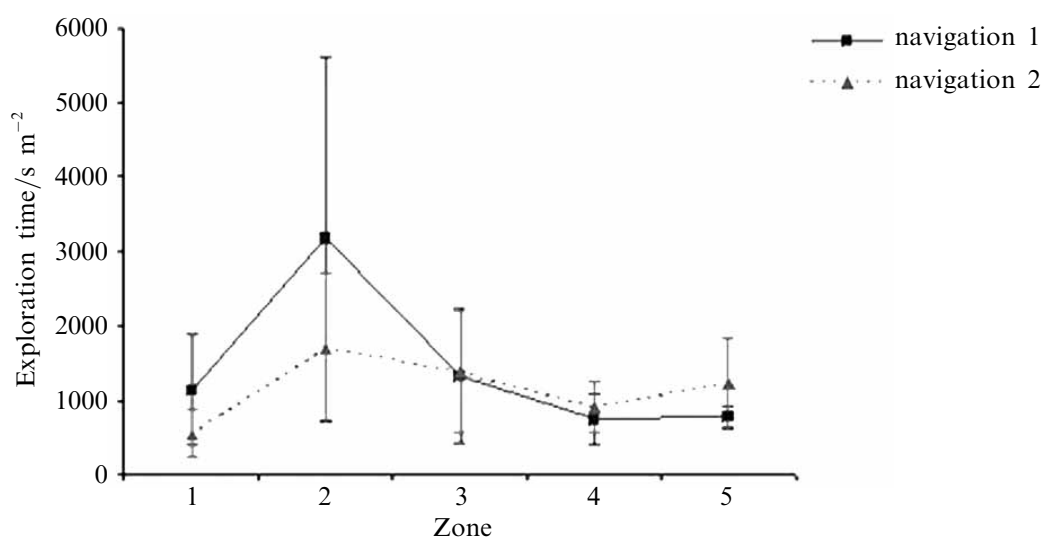

Figure 9. Evolution of average exploration time for both navigations throughout the 5 zones (with standard deviations), set to a single $1 \mathrm{~m}^{2}$ surface. 
During the first navigation, subjects needed significantly more time $(p<0.005)$ to explore zone 2, which was smaller and of a more complex configuration, than they did in the starting room, which was larger. The training process was effective because, very rapidly, from zone 2 in navigation 1, subjects took less time for exploration. This exploration time then stabilised until the maze exploration had been completed. This evolution profile was also noted in route 2 , session 4 , but to a lesser extent. In this respect, exploration time significantly decreased from zone 2 to zone 3 in navigation $1(p<0.003)$, before becoming steady. Exploration durations in zones 4 and 5 remained significantly inferior to the ones in zone 2 (respectively, $p=0.0012$ and $p<0.0013)$. In zone 2 during the first exploration, exploration time was relatively steady and the peak disappeared in navigation 2 (exploration time did not increase significantly from zone 1 to zone $2, p=0.37$ ). This observation provided evidence that the skills the subjects had acquired were maintained when they performed the second navigation (route 2, session 4). The fact that this peak disappeared also meant that the subjects needed a significantly shorter exploration time in zone 2 when performing the second navigation than in zone $1(p<0.007)$.

Correlational analyses between correct-reply rate and exploration-time per surface unit revealed that there was a negative correlation in zones 2 and 4 (respectively, $r_{1}=-0.92, p<0.05$; and $\left.r_{1}=-0.75, p<0.05\right)$ for the first route. So, the long exploration time recorded in zone 2 was correlated with a low success rate, whereas the high success rate in zone 4 was correlated with a short exploration time. These results were also noted in zone $3\left(r_{1}=-0.86, p<0.05\right)$, for the second route. A short exploration time was connected with a high correct-reply rate in zone 3 . The improvement in discrimination capacities - represented by a higher rate of correct replies - was noted because the robot moved more rapidly, at least in zones 2 and 4 throughout the first route, and in zone 3 throughout the second route.

\subsection{Subjective analysis}

4.3.1 Externalising explored cues. After analysing subjects' spontaneous testimonies collected in a questionnaire during navigation and just before the task ended, a change in terms of quality seemed to occur between navigations 1 and 2 (in routes 1 and 2). During the first navigation (route 1), subjects stated that they felt stimuli on the matrix, and their attention was being monopolised by their handling the joysticks to control the robot. During the second navigation (route 2), subjects said they perceived stimuli in space, and they often said that handling the robot felt like driving a car (cf infra). According to some subjects, when going through a door, their actions consisted in 'going through' the triangular cues which appeared 'in front of' them. However, even when navigating route 2 , whenever recognising cues required a greater concentration, some subjects once again appeared to focus their attention on tactile sensation.

4.3.2 Subjects and explored space. Thanks to the TVSS, can this feeling of 'presence' be developed (Maguire et al 1999)? Subjects experienced this feeling when the place they subjectively perceived they were in differed from the one they actually (ie physically) were in. In other words, objects could be perceived in space by subjects who felt they were themselves in the same space. They therefore felt that they were in the same space as the objects they explored. During the first exploration, all subjects felt they were in this space when they were in front of the triangular cues on the doors. During the second exploration, all subjects felt they were in the explored space when they were in front of the cues. When controlling the robot, those who felt as if they were moving like the robot had the impression they were in the place of the robot. Some said they needed to project themselves into space when they had to carry out manoeuvres. They felt, thereby, they really were in the driver's seat: "Stimulations are like intensifications which enable you to find your bearings. One imagines then they 
are in space and have to carry out movements". Subjects used many words which referred to this sense of belonging. They expressed themselves as if they did navigate within the maze and as if they did carry out manoeuvers physically. All subjects spontaneously used the word "I". The following remarks were heard: "I'm going backwards", "I'm going to turn around", "I'm reaching a cue", "I'm going forwards", "I'm stuck", "I'm turning to the right", "I'm in front of a cue", "I'm turning around", "I'm going backwards", and "I can see nothing".

4.3.3 Having access to space dimensions. Lenay (1997) studied the three types of space dimensions - height, width, and depth - that subjects could reach via a visuo-tactile sensory-substitution device. Subjects perceive a space dimension if they are capable of going back to the previous position-in other words, if their movements allow them to reverse their actions. Depth is more special than the other dimensions, because the action that leads to it generates a movement which was perceived in space. Depth requires that subjects integrate their actions as movements 'in' space (Lenay 1997). In the present experiment, most subjects stated they had access to all of the three dimensions they were asked about. It seemed that this perception was better in the second navigation. Height was mainly reported to have a connection with getting closer to the cues. It extended the tactile images of objects. Depth was perceived to have a close relation with the distance between the robot and what it was exploring. This was very often the case when subjects made forward-backward movements to order to readjust the position of the robot. This movement enabled them to make object size and stimulation intensity vary. The distance between the robot and the target was associated with the intensity of stimulation. Perceiving perspective cues collected by the robot-when going along lateral stripes in a corridor-also permitted the subjects to perceive depth.

\subsection{Comparison with vision}

Performance of blindfolded sighted subjects in a navigation task on the sole basis of visuo-tactile stimulations from the TVSS therefore appeared surprisingly good in spite of poor spatial resolution of tactile images. We wondered whether subjects could navigate efficiently with such spatial resolution in vision in order to determine whether the environment was clearly well-encoded at this resolution of $8 \times 12$ pixels or whether the tactile modality appeared more dedicated to process such poor resolution data.

The navigation capacities of blindfolded sighted subjects on the sole basis of visuotactile information were compared with the efficiency of ten sighted subjects (five females aged from 19 to 36 years, mean age: 24 years; and five males aged from 23 to 39 years, mean age: 30 years) who performed the same navigation task watching the visual monitor system of the TVSS.

Despite the poor spatial resolution of the visual images converted by the TVSS computer, subjects were able to carry out the task either in vision or with the TVSS.

Evolution curves for exploration time throughout the 5 zones of the maze (the surface of every zone being set to the same scale of a $1 \mathrm{~m}^{2}$ surface) look quite similar for the two groups (see figure 10). Nevertheless, the sighted seeing group, using the visual monitor display, showed:

(i) a significantly greater accuracy of cue identification in comparison with both the first navigation of blindfolded sighted subjects (respectively $100 \%$ versus $74 \%$; $F_{1,16}=54.76, p<0.0001$ ) and the second navigation as well (respectively $100 \%$ versus $\left.70.7 \% ; F_{1,16}=254.78, p<0.0001\right)$;

(ii) a shorter exploration time in comparison with both the first navigation of blindfolded sighted subjects (respectively, $950 \mathrm{~s}$ versus $2639 \mathrm{~s} ; F_{1,16}=20.092, p<0.0001$ ), and the second navigation as well (respectively, $950 \mathrm{~s}$ versus $3909 \mathrm{~s} ; F_{1,16}=14.5, p=0.0015$ ).

Thus, the environment is clearly sufficiently well encoded by the TVSS at a resolution of $8 \times 12$ pixels. 


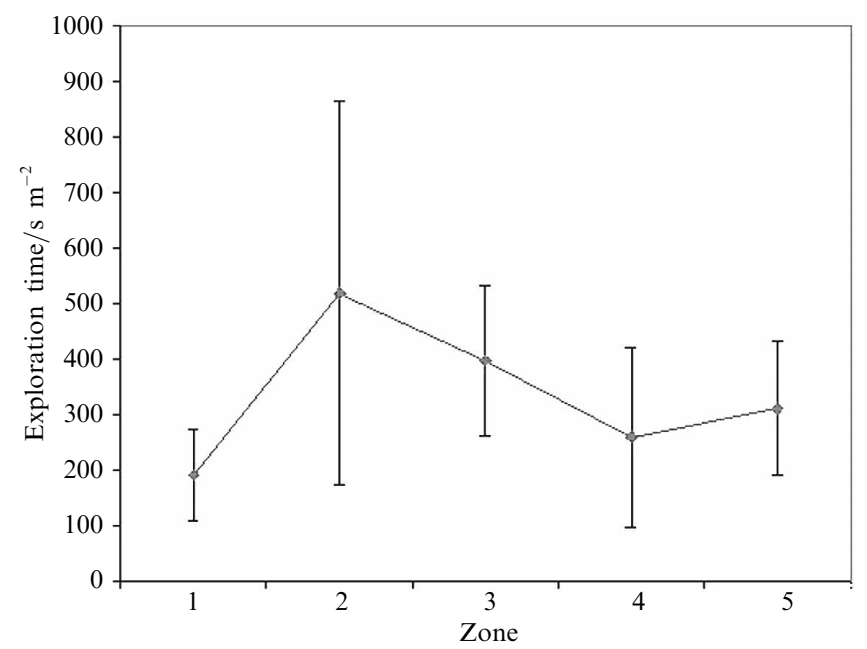

Figure 10. Evolution of average exploration time for navigation under visual control (by watching the TVSS screen) throughout the 5 zones (with standard deviations), set to a single $1 \mathrm{~m}^{2}$ surface.

\section{Discussion}

The main objective of our research was to study whether blindfolded sighted subjects would be able to carry out a spatial navigation task in a true 3-D maze, by remotely controlling a mobile robot and using visuo-tactile information. We have succeeded in revealing for the first time that it was possible for subjects to carry out such a task on the sole basis of visuo-tactile information in a limited number of sessions. As initial TVSS studies (Bach-y-Rita et al 1969; White et al 1970; Bach-y-Rita 1972; Collins et al 1973) had showed, blindfolded sighted subjects were able, from the very first trials, to detect and identify simple targets.

Once we had analysed the results, we noted a training effect at several levels.

(i) First of all, an improvement in discrimination performance during familiarisation/ assessment phases was noted as the familiarisation criterion was met faster. (ii) 2-D object-discrimination capacities, solicited in familiarisation/assessment phase, underwent a generalisation in navigation phases. Although they were often perceived while being in perspective, instead of facing the front of the robot, and being perpendicular to the TV camera, triangular cues were still recognised. This positioning distorted the visual images projected to the TVSS electrotactile array. When using a sensory-substitution device, we discovered a phenomenon similar to the one observed in visual modality. The phenomenon consisted in identifying objects, in spite of the different angles from which they were perceived, because their frontiers remained unaltered. (iii) Lastly, an improvement in the rate of correct replies (ie choices regarding cues) and exploration time from one zone to the next (set to a single $1 \mathrm{~m}^{2}$ surface unit) was observed. The difficulties subjects encountered in the first exploration seemed to have decreased during the second exploration. Furthermore, exploration time rapidly decreased from the second zone in the first exploration, before remaining steady from the third zone. In the second exploration, subjects did not lose the skills they had acquired during this training.

Our second goal was to consider whether there was a possibility that subjects performing the task subjectively experienced the perception of cues as if the latter faced them in extra-corporeal space. This phenomenon refers to the pioneer work by Bach-y-Rita and his colleagues (Bach-y-Rita 1972; Guarniero 1974, 1977), who showed that this subjective experience appeared after training with the TVSS thanks to subjects' self-initiated actions on the camera. 
According to Lenay (1997), the TVSS is a system that offers the possibility of experimentally studying the issue of consciousness of something being externalised called 'intentionality'. According to this author, perceiving things in space makes it difficult for subjects to consider (or master) the simultaneity of various events by, once again, feeling the same sensations they had experienced in a previous 'position' after returning to it with a 'gaze'.

The subjects' testimonies in this experiment suggested an externalising phenomenon did exist. When carrying out the first navigation, most subjects reported that they perceived the cues on their skin. Subsequently, during the second navigation, none of the subjects reported any longer perception of stimuli solely on the skin. They said that they subjectively experienced shapes in the space facing them.

The way subjects perceived themselves in relation to the space they were exploring increased their feeling about their externalising cues. Very soon, subjects felt involved in the space they were exploring when in front of the cues, as well as when exploring the maze environment. While carrying out the route in the second navigation, subjects were 'inside' the maze just like the robot. During the second navigation, depth was perceived more intensely as well. This was generated mainly by one clue, such as the apparent size of triangular cues and lateral stripes. Subjects interpreted decreasing tactile images on the matrix as moving-away cues-and, conversely, increasing tactile images as moving-closer cues to be a consequence of their actions; they did not merely perceive the decrease or increase of shapes.

Although the robot receiver physically explored space and thereby supplied visual information, subjects felt they were involved in this space. This feeling made us question the nature of spatialisation in subjects. This navigation task was carried out via TVSS information. So, did this task enable subjects to mentally build a space by exploring the maze step-by-step and discriminating the cues via the robot, or did subjects try and rebuild a space on the basis of a representation they had elaborated from the exploration carried out under visual control in session 2? This question, which involves specialisation genesis, will be partly answered with the most recent studies in progress with blindfolded sighted and congenitally blind subjects.

Lastly, subjects had a particular attachment to the upwardly opening triangular cues. When recognising them, subjects explicitly expressed their jubilation: "That's it, I've made it!" "It's just in front of me!" "It's the right door!". This cue category represented the item target they had to find. It was the one that was required to be correctly identified in order to progress in the navigation. One could question whether in the process of this task, via the particular connotation such a cue became linked to, subjects acceded to the qualia of sensory experience, in terms of affection quality of tactile stimulation (cf Bach-y-Rita 1995, 1997; Bach-y-Rita et al 2003). As Bach-y-Rita had already pointed out, experiencing these qualia may suggest that it is difficult to accede even to this emotional content for TVSS-equipped subjects-in particular for the early blind. This emotional content can, however, occur in the experiencing of sensory substitution in certain conditions connected to the context. This affection value was associated with a particular kind of stimulation, according to the particular meaning subjects gave it when performing exploration, such as a key allowing them to carry on their way. Even though speculative, this hypothesis offers prospects for important studies which could lead to therapeutic, educational, and game applications involving sensory-substitution devices oriented towards visually impaired subjects.

Performance made possible by these devices remained dependent on technological improvements (such as miniaturisation and video resolution) and on efficient training procedures in order to be self-sufficiently used in everyday-life environment. Our current studies with blindfolded sighted and congenitally blind subjects have continued with a large number of electro-tactile and vibro-tactile sensory-substitution 'human machine 
interfaces' (HMI) developed and applied to various surface areas. The tongue, in particular, provides a practical HMI (very sensitive and highly mobile). These devices can be used by persons with visual damage (they learn to make perceptual judgments using visual means of interpretation such as perspective, parallax, zooming, and depth estimates) or with bilateral vestibular damage (using a head-mounted accelerometer and an electro-tactile HMI through the sensory receptors of the tongue, allowing them to re-establish head-postural control). And last but not least, behavioural data on visual information perception by tactile modality are to be completed soon by a complementary behavioural and neuroimaging (fMRI and PET study) approach to cerebral changes and brain plasticity due to intensive training with visuo-tactile devices in blindfolded sighted and blind subjects. This study will provide some essential additional data for understanding sensory substitution, spatialisation, and externalisation phenomena.

Acknowledgments. This research was supported by Grant INSERM/CNAMTS no. 3 AM 077 and European Community Grant 'SENSUB' no. QoLG3-CT-2000-01797. We gratefully acknowledge Vincent Chétiennot, Etienne Lelièvre, Nathalie Vaillant, and Walter Wolf for their assistance and/or technical aid.

\section{References}

Aiello G L, 1998 "Multidimensional electrocutaneous stimulation" IEE Transactions on Rehabilitation Engineering $695-101$

Arias C, 1996 "L'écholocation humaine chez les handicapés visuels" L'Année Psychologique 96 $703-721$

Arno P, Capelle C, Wanet-Defalque M-C, Catalan-Ahumada M, Veraart C, 1999 "Auditory coding of visual patterns for the blind" Perception 28 1013-1029

Ashmead D H, Hill E W, Talor C R, 1989 "Obstacle perception by congenitally blind children" Perception \& Psychophysics $46425-433$

Bach-y-Rita P, 1972 Brain Mechanisms in Sensory Substitution (New York: Academic Press)

Bach-y-Rita P, 1995 Nonsynaptic Diffusion Neurotransmission and Late Brain Reorganization (New York: Demos-Vermande)

Bach-y-Rita P, 1997 "Substitution sensorielle et qualia", in Perception et intermodalité: Approches actuelles de la question de Molyneux Ed. J Proust (Paris: Presses Universitaires de France)

Bach-y-Rita P, 1999 "Theoretical aspects of sensory substitution and of neurotransmitter-related reorganization in spinal cord injury" Spinal Cord $37465-474$

Bach-y-Rita P, Collins C C, Saunders F, White B, Scadden L, 1969 "Vision substitution by tactile image projection" Nature $221963-964$

Bach-y-Rita P, Kaczmarek K A, Mitchell E T, 2003 "A tongue-based display for portrayal of environmental characteristics", in Psychological Issues in the Design and Use of Virtual and Adaptative Environments Eds L Hettlinger, M Haas (Mahwah, NJ: Lawrence Erlbaum Associates) pp $169-186$

Berthoz A, 1997 Le sens du mouvement (Paris: Edition Odile Jacob)

Blasch B, Wiener W, Welsh R, 1997 Foundations of Orientation and Mobility second edition (New York: American Foundation for the Blind) pp 291-293

Collins C C, Bach-y-Rita P, Saunders F, White B, Scadden L, 1973 "Transmission of pictorial information through the skin" Advances in Biological Medicine and Physiology 14285 - 315

Durup M, 1985 "Les mécanismes de l'orientation lointaine chez l'animal", in La lecture sensorimotrice et cognitive de l'expérience spatiale volume 1, Ed. J Paillard (Paris: CNRS) pp $181-190$

Farcy R, Damaschini R, Milchberg N, Sampaio E, Brenière Y, Maton B, 1997 "A laser profilometer as a space perception system for blind people" Journal of Optics 28 207-213

Flitman S, O'Grady J, Cooper V, Grafman J, 1997 "PET imaging of maze processing” Neuropsychologia $35409-419$

Foulke E, 1982 "Perception, cognition, and the mobility of blind pedestrians" Spatial Abilities. Development and Physiological Foundations (New York: Academic Press) 55-76

Gibson J J, 1966 The Senses Considered as Perceptual Systems (Boston, MA: Houghton Mifflin)

Gibson J J, 1979 The Ecological Approach to Visual Perception (Boston, MA: Houghton Mifflin)

Griffin D B, 1942 "Bird navigation" Biological Reviews $27359-400$

Guarniero G, 1974 "Experience of tactile vision" Perception $3101-104$

Guarniero G, 1977 "Tactile vision: a personal view" Journal of Visual Impairment \& Blindness 71 $125-130$

Held R, Durlach N, 1992 “Telepresence” Presence 1 109-112 
Jansson G, 1983 "Tactile guidance of movement" International Journal of Neuroscience 19 $37-45$

Kaczmarek K, Bach-y-Rita P, 1995 "Tactile displays", in Virtual Environments and Advanced Interface Design Eds W Barfield, T Furness III (Oxford: Oxford University Press) pp 349-414

Kay N, 1980 "Reaching to sound" Journal of Visual Impairment \& Blindness 74 163-166

Lenay C, 1997 "Mouvement et perception: médiation technique et constitution de la spatialisation", in Le mouvement. Des boucles sensori-motrices aux représentations cognitives et langagières Actes de la Sixième École d'Été de l'Association pour la Recherche Cognitive, pp 69-80

Lenay C, Gapenne O, Hanneton S, Marque C, Genouëlle C, 2000 "La substitution sensorielle: limites et perspectives", in Toucher pour connaitre; Psychologie cognitive de la perception tactile manuelle Eds Y Hatwell, A Streri, E Gentaz (Paris: Presses Universitaires de France) pp 287-306

Lepecq J-C, Pêcheux M-G, 1985 "L'espace géographique: inférences et représentations spatiales", in La lecture sensori-motrice et cognitive de l'expérience spatiale volume 1, Ed. J Paillard (Paris: CNRS) pp $191-201$

Maguire E A, Burgess N, O'Keefe J, 1999 "Human spatial navigation: cognitive maps, sexual dimorphism, and neural substrate" Current Opinion in Neurobiology $9171-179$

Morgan M J, 1977 Molyneux's Question (Cambridge: Cambridge University Press)

Morris R G M, 1981 "Spatial localization does not require the presence of local cues" Learning and Motivation 12 239-260

Pacherie E, 1997 "Du problème de Molyneux au problème de Bach-y-Rita", in Perception et intermodalité. Approches actuelles de la question de Molyneux Ed. J Proust (Paris: Presses Universitaires de France) pp 255-293

Proust J, 1997 Perception et intermodalité. Approches actuelles de la question de Molyneux (Paris: Presses Universitaires de France)

Sampaio E, 1989a "La vision est-elle nécessaire pour apprendre à marcher? Étude préliminaire et approche méthodologique" Psychologie Française 34 71-78

Sampaio E, 1989b "Is there a critical age for using the sonicguide with blind infants?" Journal of Visual Impairment \& Blindness $83105-108$

Sampaio E, 1995 "Les substitutions sensorielles adaptées aux déficits visuels importants", in Le déficit visuel. Des fondements neurophysiologiques à la pratique de la réadaptation Eds A B Safran, A Assimacopoulos (Paris: Masson) pp 197-211

Sampaio E, 2000 La peau peut-elle remplacer l'xil en cas de cécité? A research report (Strasbourg, France: INSERM/CNAMTS)

Sampaio E, Dufier J L, 1988 "Suppléance sensorielle électronique pour les jeunes enfants aveugles" Journal Français d'Ophthalmologie $11161-167$

Sampaio E, Maris S, Bach-y-Rita P, 2001 "Brain plasticity: 'Visual' acuity of blind persons via the tongue" Brain Research $908204-207$

Silverstone B, Lang M A, Rosenthal B P, Faye E E, 2000 The Lighthouse Handbook on Vision Impairment and Vision Rehabilitation (New York: The Lighthouse and Oxford University Press)

Van Horn J D, Gold J M, Esposito G, Ostrem J L, Mattay V, Weinberger D R, Berman K F, 1998 "Changing patterns of brain activation during maze learning" Brain Research 793 29-38

Warren D H, Strelow E R (Eds), 1985 Electronic Spatial Sensing for the Blind (Dordrecht, The Netherlands: Martinus Nijhoff)

White B W, Saunders F A, Scadden L, Bach-y-Rita P, Collins C C, 1970 "Seeing with the skin" Perception \& Psychophysics $723-27$

Witmer B G, Singer M J, 1994 Measuring Presence in Virtual Environments. ARI Technical Report 1014 (Alexandria, VA: US Army Research Institute for the Behavioral and Social Sciences)

Wysocki D, 2000 http://www.forethoughtdevelopment.com/videotac.html 


\section{PERCEPTION}

www.perceptionweb.com

This article is an advance online publication. It will not change in content under normal circumstances but will be given full volume, issue, and page numbers in the final PDF version, which will be made available shortly before production of the printed version.

Conditions of use. This article may be downloaded from the Perception website for personal research by members of subscribing organisations. Authors are entitled to distribute their own article (in printed form or by e-mail) to up to 50 people. This PDF may not be placed on any website (or other online distribution system) without permission of the publisher. 